\title{
Singularity-invariant leg rearrangements in Stewart-Gough platforms
}

\author{
Júlia Borràs, Federico Thomas and Carme Torras \\ Institut de Robòtica i Informàtica Industrial (CSIC-UPC), \\ Llorens Artigas 4-6, 08028 Barcelona, Spain. \\ e-mail: \{jborras, fthomas, ctorras\}@iri.upc.edu
}

\begin{abstract}
This work presents a necessary and sufficient condition to define a singularity-invariant leg rearrangement, based on an affine relation between the squared leg lengths before and after the rearrangement. This condition is then specified for four rigid components that can occur in StewartGough platforms, leading to the characterization of singularity-invariant leg rearrangements on all of them.
\end{abstract}

Key words: Stewart-Gough platform, Forward Kinematics, Singularities.

\section{Introduction}

The Stewart-Gough platform is defined as a 6-DoF parallel mechanism with six identical SPS legs $[1,2]$. It has remained one of the most widely studied because, despite its geometric simplicity, its analysis translates into challenging mathematical problems. One important part of this analysis corresponds to the characterization of its singularities, which has only been completely solved for some specializations (for example, designs in which some spherical joints coalesce to form multiple spherical joints [3, 4]).

Finding leg rearrangements in a given Stewart-Gough platform that leave the singularity locus invariant does not solve the problem of characterizing singularities, but it provides a lot of insight that proves useful in several ways. For example, such leg rearrangements permit simplifying the platforms geometry to ease the task of obtaining the sought characterization of its singularity locus. On the other hand, if this locus is already characterized, modifying the placement of legs permits improving some platform characteristics (such as stiffness, avoidance of leg collisions or elimination of multiple spherical joints) without altering such locus. Eventually, by analyzing all possible leg rearrangements, one could identify all equivalent platforms.

In addition, singularity-invariant leg rearrangements provide a straightforward characterization of architectural singularities, promising a common framework to the extensive literature on this topic $[5,6,7,8,9]$. 

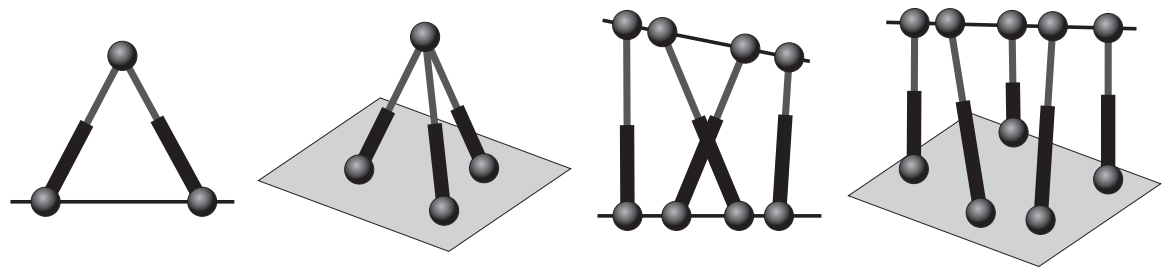

Fig. 1 The four possible rigid components involving linear geometric elements in Stewart-Gough platforms.

Now, let us suppose that we would like to apply a singularity-invariant leg rearrangement limited to a subset of legs. Clearly, this is only possible if this subset of legs defines a rigid subassembly. Kong and Gosselin refer to these subassemblies as components [10]. The simplest component arises when two legs share an attachment. The result is called the Point-Line component. Similarly, the three other components involving linear geometric entities (points, lines and planes) are the Point-Plane, Line-Line and Line-Plane components (Fig. 1).

Leg rearrangements were previously proposed by the authors for each of these components $[11,12,13]$. In this work a common framework for them all is provided in this way: all are shown to satisfy the same necessary and sufficient condition, confirming, through a unifying approach, that such rearrangements are indeed singularity-invariant.

This paper is organized as follows: Section 2 introduces the condition for singularity invariance. Then, in Sections 3 to 6 , this condition is applied to each of the components in Fig. 1. Finally, Section 7 summarizes the main results and points out some future research directions.

\section{Condition for singularity invariance}

For a general Stewart-Gough platform, the linear actuators' velocities, $\dot{l}_{1}, \dot{l}_{2}, \ldots, \dot{l}_{6}$, can be expressed in terms of the platform velocity vector $(\mathbf{v}, \Omega)$ as follows:

$$
\operatorname{diag}\left(l_{1}, \ldots, l_{6}\right)\left(\begin{array}{c}
\dot{l}_{1} \\
\dot{l}_{2} \\
\vdots \\
i_{6}
\end{array}\right)=\mathbf{J}\left(\begin{array}{c}
\mathbf{v} \\
\Omega
\end{array}\right)
$$

where $\mathbf{J}$ is the matrix of normalized Plücker coordinates of the six leg lines [1]. The parallel singularities of the platform are those configurations in which $\operatorname{det}(\mathbf{J})=0$ [14].

Now, let us change the location of the leg attachments so that the lengths of the legs in their new locations, say $d_{1}, d_{2}, \ldots, d_{6}$, are related to those of the original legs, 
$l_{1}, l_{2}, \ldots, l_{6}$, through the relation:

$$
\left(\begin{array}{c}
d_{1}^{2} \\
d_{2}^{2} \\
\vdots \\
d_{6}^{2}
\end{array}\right)=\mathbf{A}\left(\begin{array}{c}
l_{1}^{2} \\
l_{2}^{2} \\
\vdots \\
l_{6}^{2}
\end{array}\right)+\mathbf{b}
$$

where $\mathbf{A}$ and $\mathbf{b}$ are a constant matrix and a constant vector, respectively. Differentiating this equation with respect to time and substituting (1) in the result, we get

$$
\operatorname{diag}\left(d_{1}, \ldots, d_{6}\right)\left(\begin{array}{c}
\dot{d}_{1} \\
\dot{d}_{2} \\
\vdots \\
\dot{d}_{6}
\end{array}\right)=\mathbf{A J}\left(\begin{array}{c}
\mathbf{v} \\
\Omega
\end{array}\right)
$$

Then, the singularities of the platform after the leg rearrangement leading to (2) are those configurations in which $\operatorname{det}(\mathbf{A J})=\operatorname{det}(\mathbf{A}) \operatorname{det}(\mathbf{J})=0$. If $\operatorname{det}(\mathbf{A}) \neq 0$, the leg rearrangement is said to be singularity-invariant. If $\operatorname{det}(\mathbf{A})=0$, the rearrangement introduces an architectural singularity, i.e., the resulting platform is always in a singularity independently of its leg lengths [5].

Since lengths are assumed to be positive magnitudes, equation (2) defines a oneto-one relationship between leg lengths before and after a singularity-invariant leg rearrangement. As a consequence, this kind of transformations leaves not only the singularities of the platform unaltered, but also the nature and number of its assembly modes.

\section{Point-Line component}

A general leg rearrangement on the Point-Line component consists in the substitution of any leg by another one going from the point to the line (gray leg on Fig. 2-left). Considering the new leg length $d$, the following relation was proved in [12] using Heron's tetrahedron volume formula:

$$
d^{2}=\frac{n l_{1}^{2}+m l_{2}^{2}-m n}{m+n} .
$$

As this is an affine relation, following Section 2 we can state that any leg rearrangement within a Point-Line component leaves singularities invariant. 

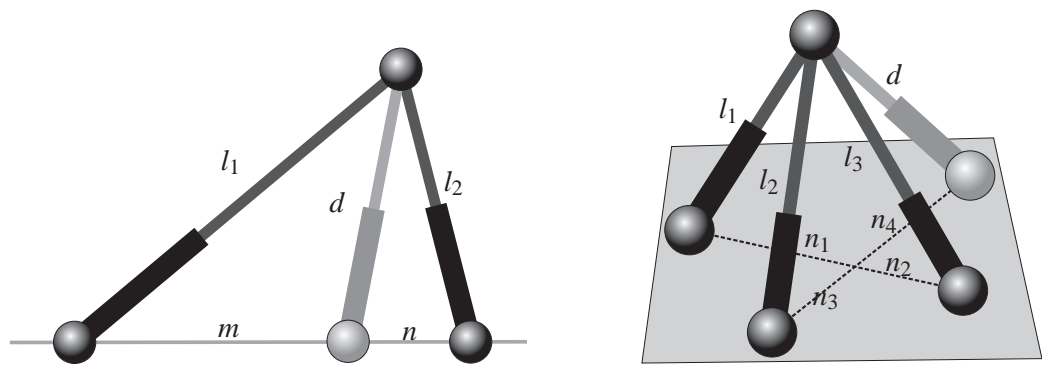

Fig. 2 A Point-Line component (left) and a Point-Plane component (right).

\section{Point-Plane component}

Proceeding similarly, let us substitute a leg of the Point-Plane component by another one going from the vertex of the tripod to any point on the base plane (Fig. 2-right). The tripod contains three Point-Line components, so equation (4) can be used twice to obtain the following affine relation between the new leg length $d$ and $l_{1}, l_{2}$ and $l_{3}$ :

$$
d^{2}=\frac{n_{2}\left(n_{3}+n_{4}\right)}{n_{3}\left(n_{1}+n_{2}\right)} l_{1}^{2}-\frac{n_{4}}{n_{3}} l_{2}^{2}+\frac{n_{1}\left(n_{3}+n_{4}\right)}{n_{3}\left(n_{1}+n_{2}\right)} l_{3}^{2}-\frac{n_{1} n_{2}\left(n_{3}+n_{4}\right)}{n_{3}}+n_{4}\left(n_{3}+n_{4}\right) .
$$

As a result, we can state that any leg rearrangement within a Point-Plane component leaves singularities invariant.

\section{Line-Line component}

Following the notation introduced in Fig. 3-left, suppose that we want to compute the length of a new leg between a point on the base line $\mathbf{a}=(x, 0,0)$ and a point on the platform line $\mathbf{b}=\mathbf{p}+z \mathbf{i}$.

Taking the distance equations of the four legs $l_{i}^{2}=\left\|\mathbf{b}_{i}-\mathbf{a}_{i}\right\|^{2}$, for $i=1, \ldots, 4$, together with that of the new leg $d^{2}=\|\mathbf{b}-\mathbf{a}\|^{2}$, the subtraction of the equation $u^{2}+v^{2}+w^{2}=1$ cancels all quadratic terms in $u, v$ and $w$, yielding

$$
\begin{aligned}
& z_{i} t-x_{i} p_{x}-x_{i} p_{z} u+\frac{1}{2}\left(p_{x}^{2}+p_{y}^{2}+p_{z}^{2}+x_{i}^{2}+z_{i}^{2}-l_{i}^{2}\right)=0, \text { for } i=1, \ldots, 4 \\
& z t-x p_{x}-x p_{z} u+\frac{1}{2}\left(p_{x}^{2}+p_{y}^{2}+p_{z}^{2}+x^{2}+z^{2}-d^{2}\right)=0
\end{aligned}
$$

where $t=\mathbf{p} \cdot \mathbf{i}$. In addition, subtracting the first equation from the others, quadratic terms in $p_{x}, p_{y}$ and $p_{z}$ cancel too, and the system becomes linear: 

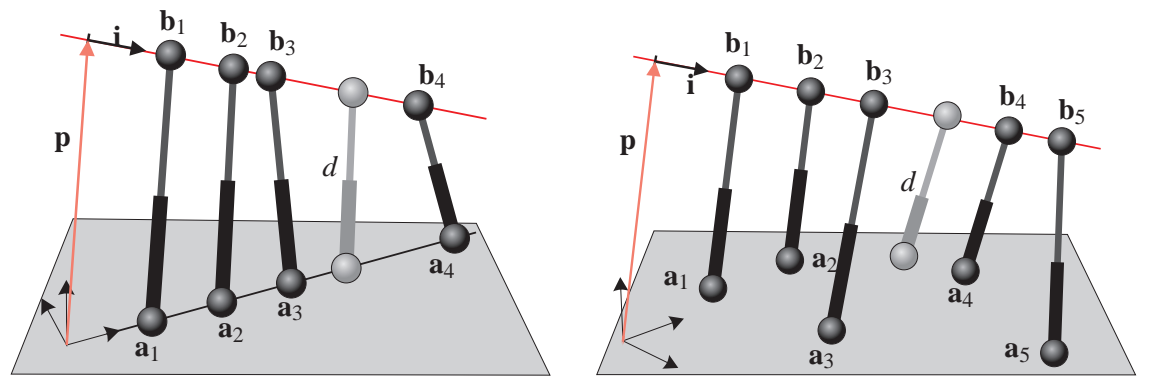

Fig. 3 On the base reference frame, local coordinates of the base attachments are $\mathbf{a}_{i}=\left(x_{i}, 0,0\right)$ on the Line-Line component (left), and $\mathbf{a}_{i}=\left(x_{i}, y_{i}, 0\right)$ on the Line-Plane component (right). In both cases, the pose of the upper line with respect to the base plane can be described by the position vector $\mathbf{p}=\left(p_{x}, p_{y}, p_{z}\right)^{T}$ and the unit director vector of the line $\mathbf{i}=(u, v, w)^{T}$. Thus, the coordinates of the leg attachments in the platform line, expressed in the base reference frame, can be written as $\mathbf{b}_{i}=\mathbf{p}+z_{i} \mathbf{i}$, for $i=1, \ldots, 4$ (left) and $i=1, \ldots, 5$ (right).

$$
\left(\begin{array}{cccc}
z_{1}-z_{2} & x_{2}-x_{1} & x_{2} z_{2}-x_{1} z_{1} & 0 \\
z_{1}-z_{3} & x_{3}-x_{1} & x_{3} z_{3}-x_{1} z_{1} & 0 \\
z_{1}-z_{4} & x_{4}-x_{1} & x_{4} z_{4}-x_{1} z_{1} & 0 \\
z_{1}-z & x-x_{1} & x_{z}-x_{1} z_{1} & \frac{1}{2}
\end{array}\right)\left(\begin{array}{c}
t \\
p_{x} \\
u \\
d^{2}
\end{array}\right)=\left(\begin{array}{c}
N_{2} \\
N_{3} \\
N_{4} \\
N
\end{array}\right)
$$

where $N_{i}=1 / 2\left(x_{i}^{2}+z_{i}^{2}-l_{i}^{2}-x_{1}^{2}-z_{1}^{2}+l_{1}^{2}\right), i=1, \ldots, 4$, and $N=1 / 2\left(x^{2}+z^{2}-x_{1}^{2}-\right.$ $\left.z_{1}^{2}+l_{1}^{2}\right)$ are constant. Now the expression for $d^{2}$ can be obtained by solving the system using Cramer's rule:

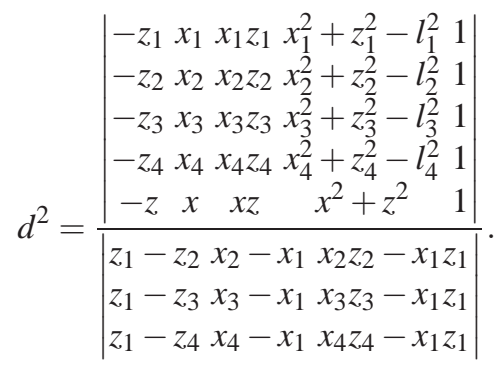

For any non-architecturally singular Line-Line component, the denominator is different from zero (in accordance with the condition found in [11]). Expanding the determinants involved in equation (7) leads to the affine relation

$$
d^{2}=c_{1} l_{1}^{2}+c_{2} l_{2}^{2}+c_{3} l_{3}^{2}+c_{4} l_{4}^{2}+c_{0},
$$

where all the coefficients depend on known constant coordinates. Thus, we can also state that any leg rearrangement within a Line-Line component leaves singularities invariant. 


\section{Line-Plane component}

Finally, let us consider the 5-legged parallel platform appearing in Fig. 3-right. We proceed in a similar way as for the Line-Line component following the notation on the figure. The system derived from leg lengths has now 5 equations plus the one for the additionally introduced leg. After the same simplifications, the following linear system is obtained:

$$
\left(\begin{array}{ccccc}
x_{2}-x_{1} & y_{2}-y_{1} & x_{2} z_{2}-x_{1} z_{1} & y_{2} z_{2}-y_{1} z_{1} & 0 \\
x_{3}-x_{1} & y_{3}-y_{1} & x_{3} z_{3}-x_{1} z_{1} & y_{3} z_{3}-y_{1} z_{1} & 0 \\
x_{4}-x_{1} & y_{4}-y_{1} & x_{4} z_{4}-x_{1} z_{1} & y_{4} z_{4}-y_{1} z_{1} & 0 \\
x_{5}-x_{1} & y_{5}-y_{1} & x_{5} z_{5}-x_{1} z_{1} & y_{5} z_{5}-y_{1} z_{1} & 0 \\
x-x_{1} & y-y_{1} & x z-x_{1} z_{1} & y z-y_{1} z_{1} & \frac{1}{2}
\end{array}\right)\left(\begin{array}{c}
p_{x} \\
p_{y} \\
u \\
v \\
d^{2}
\end{array}\right)=\left(\begin{array}{c}
\left(z_{2}-z_{1}\right) t+N_{2} \\
\left(z_{3}-z_{1}\right) t+N_{3} \\
\left(z_{4}-z_{1}\right) t+N_{4} \\
\left(z_{5}-z_{1}\right) t+N_{5} \\
\left(z-z_{1}\right) t+N
\end{array}\right)
$$

where now $N_{i}=1 / 2\left(x_{i}^{2}+y_{i}^{2}+z_{i}^{2}-l_{i}^{2}-x_{1}^{2}-y_{1}^{2}-z_{1}^{2}+l_{1}^{2}\right), i=1, \ldots, 5$, and $N=$ $1 / 2\left(x^{2}+y^{2}+z^{2}-x_{1}^{2}-y_{1}^{2}-z_{1}^{2}+l_{1}^{2}\right)$. We can always find a system matrix with a non-zero determinant for any non-architecturally singular Line-Plane component (see [13] for details).

Thus, using Cramer's rule again yields

$$
d^{2}=\frac{2(r t+s)}{C}
$$

where $C$ is the determinant of the matrix in (9) and $r$ and $s$ are the determinants

$$
r=\left|\begin{array}{ccccc}
x_{2}-x_{1} & y_{2}-y_{1} & x_{2} z_{2}-x_{1} z_{1} & y_{2} z_{2}-y_{1} z_{1} & z_{2}-z_{1} \\
x_{3}-x_{1} & y_{3}-y_{1} & x_{3} z_{3}-x_{1} z_{1} & y_{3} z_{3}-y_{1} z_{1} & z_{3}-z_{1} \\
x_{4}-x_{1} & y_{4}-y_{1} & x_{4} z_{4}-x_{1} z_{1} & y_{4} z_{4}-y_{1} z_{1} & z_{4}-z_{1} \\
x_{5}-x_{1} & y_{5}-y_{1} & x_{5} z_{5}-x_{1} z_{1} & y_{5} z_{5}-y_{1} z_{1} & z_{5}-z_{1} \\
x-x_{1} & y-y_{1} & x z-x_{1} z_{1} & y z-y_{1} z_{1} & z-z_{1}
\end{array}\right|
$$

and

$$
s=\left|\begin{array}{ccccc}
x_{2}-x_{1} & y_{2}-y_{1} & x_{2} z_{2}-x_{1} z_{1} & y_{2} z_{2}-y_{1} z_{1} & N_{2} \\
x_{3}-x_{1} & y_{3}-y_{1} & x_{3} z_{3}-x_{1} z_{1} & y_{3} z_{3}-y_{1} z_{1} & N_{3} \\
x_{4}-x_{1} & y_{4}-y_{1} & x_{4} z_{4}-x_{1} z_{1} & y_{4} z_{4}-y_{1} z_{1} & N_{4} \\
x_{5}-x_{1} & y_{5}-y_{1} & x_{5} z_{5}-x_{1} z_{1} & y_{5} z_{5}-y_{1} z_{1} & N_{5} \\
x-x_{1} & y-y_{1} & x z-x_{1} z_{1} & y z-y_{1} z_{1} & N
\end{array}\right|
$$

Note that this is not an affine relation because it depends on $t$. However, if we impose $r=0$, then the resulting expression can be rewritten as

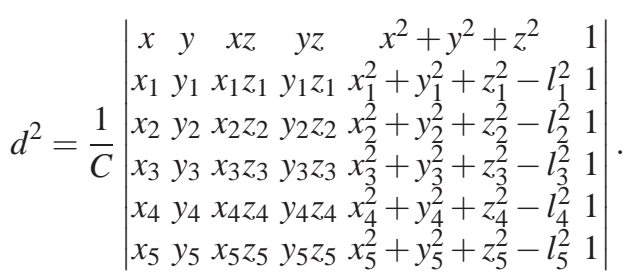




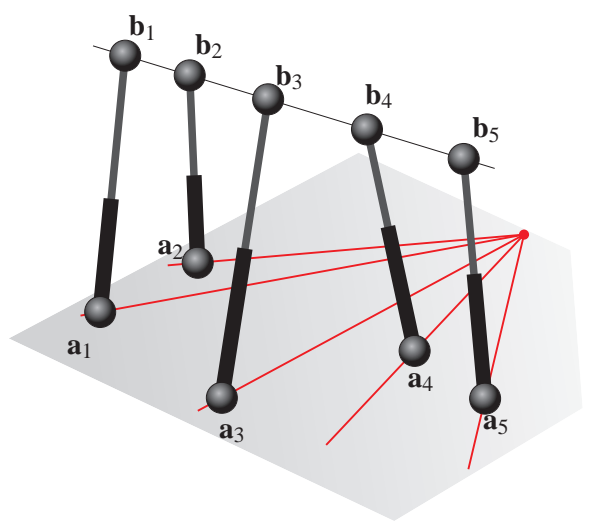

Fig. 4 Singularity-invariant leg rearrangements in the Line-Plane component must satisfy equation $r=0$, where $r$ is defined in equation (11).

After Laplace expansion by the elements of the 5th column, (13) leads to the affine relation

$$
d^{2}=c_{1} l_{1}^{2}+c_{2} l_{2}^{2}+c_{3} l_{3}^{2}+c_{4} l_{4}^{2}+c_{5} l_{5}^{2}+c_{0},
$$

where again all the coefficients depend on known constant coordinates.

In conclusion, the Line-Plane component is the first for which a general leg rearrangement is not necessarily singularity-invariant. To be so, the new leg attachments $\mathbf{a}=(x, y, 0)$ and $\mathbf{b}=\mathbf{p}+z \mathbf{i}$ must satisfy the equation $r=0$.

Specific geometric rules to perform singularity-invariant leg rearrangements can be obtained from the equation $r=0$. Indeed, it defines a one-to-one correspondence between points on the platform line and lines of a pencil on the base plane (Fig. 4). Thus, base attachments can always be moved within their corresponding lines. Furthermore, the vertex of the base pencil plays an important role in the characterization of the kinematics and singularities of the Line-Plane component (see [13] and [15] for details).

\section{Conclusions and future work}

In this paper, a necessary and sufficient condition for a leg rearrangement in a Stewart-Gough platform to preserve its singularity locus has been derived. As long as an affine relation holds between the squared leg lengths before and after the rearrangement, the platform singularities remain unchanged. In other words, a leg rearrangement is singularity-invariant if, and only if, it induces an affine mapping in the joint space of the manipulator.

It has been shown that all leg rearrangements in the Point-Line, Point-Plane and Line-Line components of a Stewart-Gough platform (excluding those leading to an architecturally-singular configuration) satisfy this condition, therefore all being 
singularity-invariant. In the case of the Line-Plane component, the condition holds only for a subset of leg rearrangements that has a neat geometric interpretation as previously shown. Thus, the present paper provides a common framework where the different singularity-preserving leg transformations proposed by the authors in previous works are viewed in a unified way.

As regards to future research, work on the double-planar Stewart-Gough platform is currently under way, suggesting interesting results for the general classification of all Stewart-Gough platforms.

It has also been briefly shown how the condition $\operatorname{det}(\mathbf{A})=0$ in Section 2 can characterize architectural singularities, thus work in this direction is in progress.

Acknowledgements This work has been partially supported by the Spanish Ministry of Education and Science, under the I+D project DPI2007-60858, and the Generalitat de Catalunya through the VALTEC program, cofinanced by FEDER funds.

\section{References}

1. Merlet, J.-P., Parallel Robots. Springer, (2000)

2. Dasguptaa, B., Mruthyunjayab, T.: The Stewart platform manipulator: a review. Mech. Mach. Theory, 35, 15-40 (2000)

3. Downing, D., Samuel, A., Hunt, K.:Identification of the special configurations of the octahedral manipulator using the pure condition: Int. J. Robot. Res. 21, 147-159 (2002)

4. Alberich-Carramiñana, M., Thomas, F., Torras, C.:Flagged parallel manipulators. IEEE Trans. Robot. 23(5), 1013-1023 (2007)

5. Ma, O., Angeles, J.: Architecture singularities of platform manipulators. In: IEEE Int. Conf. Robot. Automat. 2, pp. 1542-1547 (1991)

6. Karger, A.:Architecture singular planar parallel manipulators. Mech. Mach. Theory, 38, 1149-1164 (2003)

7. Husty, M., Karger, A.:Architecture singular parallel manipulators and their self-motions. In: Int. Sym. Adv. Robot Kinematics, pp.355-364 (2000)

8. Wohlhart, K.:Synthesis of architecturally mobile double-planar platforms. In: Int. Sym. Adv. Robot Kinematics, pp. 473-482 (2002)

9. Rschel, O. Mick, S.:Characterisation of architecturally shaky platforms. In: Int. Sym. Adv. Robot Kinematics, pp. 465-474 (1998)

10. Kong, X., Gosselin, C.: Classification of 6-SPS parallel manipulators according to their components. In: Proc. ASME Des. Eng. Tech. Conf. DETC2000/MECH-14105 (2000)

11. Borràs, J., Thomas, F., Torras, C.: Architecture singularities in flagged parallel manipulators. In: IEEE Int. Conf. Robot. Automat., pp. 3844-3850 (2008)

12. Borràs, J., Thomas, F., Torras, C.: On $\Delta$-transforms. IEEE Trans. Robot. 25(6), 1225-1236 (2009)

13. Borràs, J., Thomas, F.: Kinematics of the line-plane subassembly in Stewart platforms. In: IEEE Int. Conf. Robot. Automat., pp. 4094-4099 (2009)

14. Gosselin, C. Angeles, J.: Singularity analysis of closed-loop kinematic chains. IEEE Trans. Robot. 6(3), 281-290 (1990)

15. Borràs, J., Thomas, F., Torras, C.: A family of quadratically-solvable 5-UPS parallel robots. In: IEEE Int. Conf. Robot. Automat., to appear (2010) 